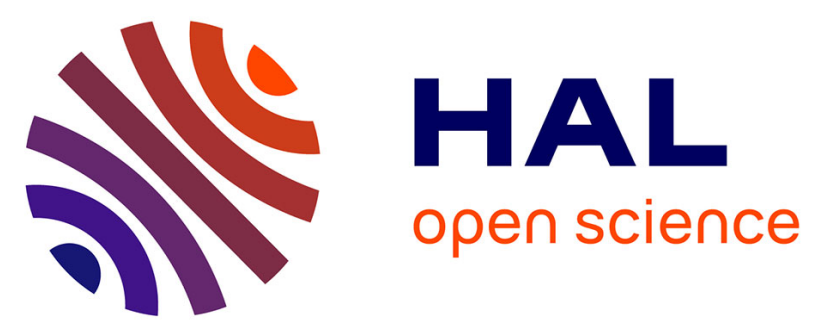

\title{
Concentration of osmoregulated periplasmic glucans (OPGs) modulates the activation level of the RcsCD RcsB phosphorelay in the phytopathogen bacteria Dickeya dadantii
}

Sébastien Bontemps-Gallo, Edwige Madec, Jacqueline Dondeyne, Brigitte Delrue, Catherine Robbe-Masselot, Olivier Vidal, Anne-France Prouvost, Gilles Boussemart, Jean-Pierre Bohin, Jean-Marie Lacroix

\section{To cite this version:}

Sébastien Bontemps-Gallo, Edwige Madec, Jacqueline Dondeyne, Brigitte Delrue, Catherine RobbeMasselot, et al.. Concentration of osmoregulated periplasmic glucans (OPGs) modulates the activation level of the RcsCD RcsB phosphorelay in the phytopathogen bacteria Dickeya dadantii. Environmental Microbiology, 2013, Plant-Microbe Interactions, 15 (3), pp.881-894. 10.1111/1462-2920.12054 . hal02133974

\author{
HAL Id: hal-02133974 \\ https://hal.science/hal-02133974
}

Submitted on 11 Feb 2020

HAL is a multi-disciplinary open access archive for the deposit and dissemination of scientific research documents, whether they are published or not. The documents may come from teaching and research institutions in France or abroad, or from public or private research centers.
L'archive ouverte pluridisciplinaire HAL, est destinée au dépôt et à la diffusion de documents scientifiques de niveau recherche, publiés ou non, émanant des établissements d'enseignement et de recherche français ou étrangers, des laboratoires publics ou privés. 


\section{Concentration of Osmoregulated Periplasmic Glucans (OPGs)}

2 modulates the activation level of the ResCD-ResB phosphorelay in

3 the phytopathogen bacteria Dickeya dadantii.

4

5 Sébastien Bontemps-Gallo ${ }^{1,2}$, Edwige Madec $^{1,2}$, Jacqueline Dondeyne $^{1,2}$, Brigitte Delrue ${ }^{2}$,

6 Catherine Robbe-Masselot ${ }^{1,2}$, Olivier Vidal $^{1,2}$, Anne-France Prouvost $^{1,2,3}$, Gilles

7 Boussemart $^{1,2,4}$, Jean-Pierre Bohin ${ }^{1,2}$, and Jean-Marie Lacroix ${ }^{* 1,2}$

8

9

${ }^{1}$ Unité de Glycobiologie Structurale et Fonctionnelle, UMR CNRS 8576, IFR 147, Université des Sciences et Technologies de Lille, 59655 Villeneuve d'Ascq, France

${ }^{2}$ Université Lille Nord de France, Lille, France

${ }^{3}$ Present address: VitamFero, 31 avenue Monge, 37200 Tours, France

${ }^{4}$ Present address: INRA Dijon, UMR 1229, 17 rue Sully, BP 86510, 21065 Dijon, France

*corresponding author. Mailing address: Université des Sciences et Technologies de Lille, Unité de Glycobiologie Structurale et Fonctionnelle, UMR CNRS 8576 IFR147, 59655

Villeneuve d'Ascq Cedex, France. Fax: +33 3204365 55. Phone: +33 3204365 92. E-mail: jean-marie.lacroix@univ-lille1.fr. 
1 Summary

2 Osmoregulated periplasmic glucans (OPGs) are general constituents of many

3 Proteobacteria. Synthesis of these oligosaccharides is repressed by increased osmolarity

4 of the medium. OPGs are important factors required for full virulence in many zoo- or

5 phytopathogens including Dickeya dadantii. The phytopathogen enterobacterium $D$.

6 dadantii causes soft-rot disease on a wide range of plant species. The total loss of

7 virulence of $o p g$-negative strains of $D$. dadantii is linked to the constitutive activation of

8 the ResCD ResB phosphorelay highlighting relationship between this phosphorelay and

9 OPGs. Here we show that OPGs control the ResCD ResB activation in a concentration

10 dependent manner, are required for proper activation of this phosphorelay by medium

11 osmolarity, and a high concentration of OPGs in planta is maintained to achieve the low

12 level of activation of the $R \operatorname{csCD} R \operatorname{csB}$ phosphorelay required for full virulence in $D$.

13 dadantii. 


\section{Introduction}

Osmoregulated periplasmic glucans (OPGs) are a family of periplasmic oligosaccharides found in the envelope of most Proteobacteria. Their two common features are that glucose is the sole constitutive sugar and their abundance in the periplasm increases as the osmolarity of the medium decreases (Bohin et al., 2006). Numerous studies indicate that these glucans belong to the common virulence factors for zoopathogens and phytopathogens Proteobacteria, since mutants devoid of OPGs exhibit a reduced or a non-virulent phenotype (Loubens et al., 1993; Mahajan-Miklos et al., 1999; Page et al., 2001; Bhagwat et al., 2009). In Enterobacteria, the glucose backbone synthesis of OPGs is catalyzed by the opgGH operon products, and opgG or opgH mutant strains are completely devoid of OPGs. OpgG and OpgH together catalyze the synthesis of linear glucans containing 5 to 12 glucose units joined by $\beta, 1-2$ linkages and branched by $\beta, 1-6$ linkages (Bohin et al., 2006).

Dickeya dadantii, an Enterobacterium, causes soft rot disease in a wide range of plant species, including many economically important crops (Perombelon et al., 2002). This opportunistic pathogen devastates crops in storage facilities or in growing plants. In $D$. dadantii, opgG or opgH mutant strains, completely devoid of OPGs, show a pleiotropic phenotype including increased synthesis of exopolysaccharides, loss of motility, induction of a general stress response and complete loss of virulence on potato tubers and chicory leaves. These phenotypes were confirmed by a proteomic analysis and suggest that strains devoid of OPGs are impaired in the perception of their environment (Page et al., 2001; Bouchart et al., 2007). The isolation of revertant mutant strains in the $\operatorname{rcs} C D B$ locus encoding a phosphorelay system, restoring several of the wild-type phenotypes, indicated that OPGs are required in the perception of environmental changes and that these glucans are needed to achieve a low level of activation of the RcsCD RcsB signaling pathway (Bouchart et al., 2010). 
1 Two component systems (also called phosphorelay) are the main systems used by bacteria to

2 sense and respond to variations of the environment. Under stimuli essentially unknown, a sensor histidine kinase autophosphorylates and transfers its phosphate group to its cognate response regulator which in turn regulates the expression of different target genes (Buelow et al., 2010).

The $\mathrm{RcsCD} R \operatorname{csB}$ phosphorelay is restricted to enterobacteria. $\mathrm{Rcs} C$ is the transmembrane kinase, RcsB the phosphorylated regulator, and phosphorylation occurs via the intermediate transmembrane protein RcsD. In several enterobacteria, it was shown that activation of the RcsCD RcsB system enhanced exopolysaccharides synthesis but also cell division, by increasing expression of the ftsAZ operon, (Carballès et al., 1999; Bouchart et al., 2010) and decreased virulence but also motility by decreasing expression of the flhDC operon (FrancezCharlot et al., 2003; Garcia-Calderon et al., 2005; Majdalani et al., 2005; Bouchart et al., 2010). The RcsCD RcsB system is induced by different stimuli including high osmolarity (Sledjeski et al., 1996; Hagiwara et al., 2003; Mouslim et al., 2003). In addition, the RcsCD RcsB phosphorelay activity is enhanced by the outer membrane lipoprotein RcsF in E. coli (Majdalani et al., 2005) and repressed by the transmembrane IgaA protein in S. enterica (Cano et al., 2002).

Data presented in this paper indicate that OPGs concentration modulate activity of the RcsCD RcsB phosphorelay. OPGs are present in bacteria throughout the virulence process in planta and are required at a minimal concentration for full virulence.

\section{Results}

Periplasmic OPGs concentration depends on arabinose concentration in the NFB3835 strain Previously, we showed that OPGs control the RcsCD RcsB phosphorelay in D. dadantii by preventing its activation and that total loss of OPGs (opgG strains) leads to a constitutive activation of this phosphorelay (Bouchart et al., 2010). Thus, it was tempting to speculate that 
1 variations of OPGs concentration affect activation of the RcsCD ResB phosphorelay. To unravel the relationship between OPGs and the RcsCD RcsB phosphorelay, we decided to test the impact of variation of OPGs concentration on the activation of the RcsCD RcsB phosphorelay.. In Escherichia coli, the $\operatorname{araBAD}$ operon is known to be tightly regulated by the concentration of L-arabinose in the medium by the AraC protein (Guzman et al., 1995). OPGs concentration synthesized by the NFB3835 strain (araC-P $\mathrm{P}_{\mathrm{BAD}}$ opg $G H$, see experimental procedures) was measured in M63 medium containing various L-arabinose concentrations ranging from $0 \mathrm{~g} \mathrm{l}^{-1}$ to $1 \mathrm{~g} \mathrm{l}^{-1}$ (Fig. 1). No OPGs could be detected without L-arabinose. OPGs concentration increased in cells as the L-arabinose concentration increased in the medium to reach a maximal concentration of $20 \mu \mathrm{g} \mathrm{mg}^{-1}$ of proteins when $1 \mathrm{~g} \mathrm{l}^{-1}$ arabinose was added to the medium. OPGs concentration remained the same when higher L-arabinose concentrations ( $2 \mathrm{~g} \mathrm{l}^{-1}$ or $5 \mathrm{~g} \mathrm{l}^{-1}$ ) were added to the medium (data not shown). This strain can be used to test relationship between OPGs and the RcsCD RcsB phosphorelay, since OPGs concentration of this strain depends on L-arabinose concentration of the medium up to $1 \mathrm{~g} \mathrm{l}^{-1}$.

Increasing concentrations of OPGs decrease the activation of the RcsCD RcsB phosphorelay

Relationship between OPGs and the RcsCD RcsB phosphorelay was followed by expression of transcriptional fusions derived from two operons known to be regulated in an opposite way by the RcsCD RcsB phosphorelay in D. dadantii (Bouchart et al., 2010). The RcsCD RcsB system regulates motility by down-regulation of the $f l h D C$ master operon encoding activators required for expression of the flagellar apparatus genes. The ftsAZ operon is needed for cell division. One of the promoters regulating this operon is up-regulated by the RcsCD RcsB phosphorelay and it was cloned in the ftsA-uidA fusion (Bouchart et al., 2010). The flhD-uidA and the ftsA-uidA fusions were transduced into the NFB3835 strain and expressions of both fusions were measured after growth in M63 medium with increasing concentrations of Larabinose. Motility was evaluated in the NFB3835 strain on M63 medium swimming plates 
1 with increasing concentrations of L-arabinose. As a control, the respective motility levels of the EC3937 (wild-type) and the NFB3723 (opgG) strains were measured in M63 medium with or without various concentrations of L-arabinose. Motility levels remained constant whatever the concentration of L-arabinose for both strains and were 2.2 fold higher for the wild-type strain as compared to the opgG mutant strain (Fig. 2A). Motility of the NFB3835 strain increased as the concentration of L-arabinose increased (Fig. 2A). The lower motility level was observed in the NFB3835 strain grown in M63 medium and was similar to the motility level observed in the NFB3723 (opgG) strain grown in the same medium. The higher motility level was observed in the NFB3835 strain grown in M63 medium with $1 \mathrm{~g} \mathrm{l}^{-1}$ arabinose added and was similar to the motility level observed in the EC3937 wild-type strain grown in the same medium (Fig. 2A). Expression of the flhD-uidA fusion was measured after growth of the NFB7300 strain in M63 medium containing the same increasing concentrations of L-arabinose (Fig. 2B). As a control, the respective $\beta$-glucuronidase activity levels of the NFB3800 (wild-type) and the NFB7301 (opgG) strains were measured in M63 medium with or without various concentrations of L-arabinose. Glucuronidase activity remained constant for both strains whatever the concentration of L-arabinose and was 2.3 fold higher in the wildtype strain as compared to the opgG mutant strain (Fig. 2B). The lower level of $\beta$ glucuronidase expression was measured after growth of the NFB7300 in M63 medium without L-arabinose and was similar to the expression level measured in the opgG strain (NFB7301) harboring the same fusion. The highest level of expression was measured after growth in M63 medium containing $1 \mathrm{~g} \mathrm{l}^{-1}$ of L-arabinose and was similar to the expression level observed in the wild-type strain harboring the same fusion (NFB3800). Increase in the motility was correlated with an increased expression of the flhD-uidA fusion. These data indicate that the concentration of OPGs modulates motility in D. dadantii. Expression of the ftsA-uidA fusion was measured in the same conditions (Fig. 2C). As a control, the respective 
1 glucuronidase activities levels of the NFB3809 (wild-type) and the NFB7274 (opgG) strains were measured in M63 medium with or without various concentrations of L-arabinose. Glucuronidase activity remained constant for both strains whatever the concentration of Larabinose and was 6 fold higher in the $\operatorname{opg} G$ strain as compared to the wild-type strain. The lower level of expression was measured after growth of the NFB3837 strain in M63 medium containing $1 \mathrm{~g} \mathrm{l}^{-1}$ L-arabinose and was similar to the expression level measured in the wildtype strain (NFB3809) harboring the same fusion. The highest level was measured after growth in M63 medium without L-arabinose and was similar to the expression level observed in the opgG strain harboring the same fusion (NFB7274). These data indicate that concentration of OPGs modulates ftsA expression in D. dadantii. To ensure that the regulation observed occurs through the RcsCD ResB phosphorelay, an $\operatorname{rcs} B$ or an $\operatorname{rcs} C$ null mutations were introduced into NFB3835, NFB3837 and NFB7300 (NFB7368, NFB7371 and NFB7369 respectively and NFB7400, NFB7406, NFB7405, respectively). Whatever the rcs genetic context, motility and glucuronidase activities of both fusions remained constant whatever the L-arabinose concentration and similar to the one observed for the wild-type strain harboring the same fusions, respectively (Fig 2A, B and C). A recent study revealed that the unphosphorylated form of RcsB is required for proper regulation of biofilm synthesis (Latasa et al. 2012). Thus, the observed phenotypes in the $\operatorname{rcs} B$ null mutant strains could be the result of the lack of the RcsB protein itself rather than the loss of phosphorylation. An $r c s C D B_{\mathrm{D} 56 \mathrm{~N}}$ locus encoding an unphosphorylatable form of $\mathrm{rcsB}$ was constructed (see experimental procedures) and introduced into the rcsB derivative strains NFB7368, NFB7369 and NFB7371 (NFB7401, NFB7403, NFB7402, respectively). Results were similar to those observed with rcsB null mutant strains for motility and glucuronidase activities (Fig. 2A, B and C). To ensure that the $\mathrm{RcsB}$ cytoplasmic concentration was independent of the Larabinose concentration, a western blot analysis was performed in M63 medium with various 
1 concentrations of L-arabinose added in the EC3937, NFB3723, NFB3835, NFB7112 and

2 NFB3682 strains. Similar amount of the RcsB protein was observed whatever the strain and

L-arabinose concentration tested (data not shown) indicating that regulation occurs through variation of the phosphorylation level of the RcsB transcriptional regulator. Taken together, these results indicate that OPGs control the RcsCD RcsB phosphorelay activity in a concentration dependent manner.

A minimal concentration of OPGs is required for $\mathrm{D}$. dadantii throughout the virulence process

The RcsCD RcsB phosphorelay activity is severely repressed during virulence (GarciaCalderon et al., 2005) and OPGs are required for this repression (Bouchart et al., 2010). Thus, OPGs concentration must affect virulence. To test the impact of OPGs concentration on virulence of $D$. dadantii during pathogenesis, NFB3835 was grown in M63 medium with various concentrations of L-arabinose prior to inoculation. Bacteria were centrifuged and washed twice with M63 medium without a carbon source to remove arabinose to avoid OPG synthesis in planta. Bacteria $\left(10^{7}\right)$ were inoculated on chicory leaves and virulence was monitored $24 \mathrm{~h}$ and $48 \mathrm{~h}$ later (Fig. 3). When no arabinose was provided to NFB3835 bacterial cells before inoculation (fig. 3), no maceration of chicory leaves was detected. This indicates that no free arabinose is available in planta. A moderate maceration of chicory leaves was observed at a concentration of $0.01 \mathrm{~g} \mathrm{l}^{-1}$ of L-arabinose while maceration similar to the wild-type strain was observed at an L-arabinose concentration of $1 \mathrm{~g} \mathrm{l}^{-1}$. Thus, because no OPG could be synthesized in planta, severity of the disease depends on OPGs concentration at time 0 and stops when OPGs concentration in bacteria falls down below the lower concentration required for virulence because of dilution of OPGs during growth of bacteria. This concentration could be estimated around $0.01 \mathrm{~g} \mathrm{l}^{-1}$ of arabinose added (Fig. 3). This Larabinose concentration corresponds to $10 \mu \mathrm{g}$ of OPGs per mg of protein (Fig. 1). When 
1 arabinose $\left(1 \mathrm{~g} \mathrm{l}^{-1}\right)$ was added to the bacterial inoculum on chicory leaves or $24 \mathrm{~h}$ after

2 inoculation, no virulence was observed when bacteria were grown in 63 medium before inoculation while full virulence was observed whatever the arabinose concentration provided in the 63 medium before inoculation (data not shown). These data indicate that a minimal level of OPGs is required during all the pathogenesis process for full virulence of $D$. dadantii and suggest that $D$. dadantii is able to adjust its OPG concentration in planta.

D. dadantii synthesizes a high concentration of OPGs in planta

To test this hypothesis, the concentration of OPGs was determined after growth of the wildtype strain in planta. The EC3937 strain was grown in M63 medium and 10 ${ }^{7}$ bacteria were inoculated on several chicory leaves. OPGs were extracted and purified from bacteria isolated from culture in M63 medium before inoculation $(0 \mathrm{~h})$, and $24 \mathrm{~h}$ and $48 \mathrm{~h}$ after growth in planta. For each time, CFU were determined. After $24 \mathrm{~h}$ of maceration, OPGs concentration increased more than twofold as compared to the concentration observed at $0 \mathrm{~h}$ and remained constant after 48 h (Fig. 4A). Because OPGs were purified on P4 gel filtration column, one can argue that OPGs extracted from bacteria grown in planta were contaminated by oligosaccharides of similar weight resulting from degradation of plant polysaccharides by hydrolytic enzymes secreted by $D$. dadantii during infection. MALDI TOF mass spectrometry was used to determine the sizes of the OPG backbones. Spectrum of OPGs extracted from bacteria grown in M63 medium was used as a control and compared with spectra of OPGs extracted from bacteria grown in planta after $24 \mathrm{~h}$ and $48 \mathrm{~h}$ of infection. As shown in Fig. 4B, no difference in the sizes of OPGs was observed, whatever growth conditions. Each of them was composed of 4 to 11 residues of glucose. This was clearly seen in mass spectra with the 8 $[\mathrm{M}+\mathrm{Na}]^{+}$molecular ions at $\mathrm{m} / \mathrm{z} 689,851,1013,1175,1337,1499,1661$ and 1823 recovered in each spectrum. For several molecular ions, an increment of 42Da was observed and corresponds to the substitution of OPGs by acetyl residues, a characteristic feature of OPGs 
1 isolated from $D$. dadantii (Cogez et al., 2001). Thus, oligosaccharides extracted from bacteria

grown in planta were only constituted of OPGs. When bacteria were grown in media of higher $(0.1$ or $0.2 \mathrm{M} \mathrm{NaCl}$ added) osmolarity or in medium of lower osmolarity (half-strength M63), no difference in the severity of the disease nor in the apparition of the symptoms were observed (data not shown). Taken together, these results indicate that in planta, D. dadantii adjust its OPG concentration to a higher level than the minimal concentration required to reach a sufficient repression level of the $\mathrm{R} c s C D \mathrm{R} c s B$ phosphorelay needed for full virulence of D. dadantii.

The RcsCD RcsB phosphorelay exhibits a low activity level during virulence

This high concentration of OPGs must be related to a low activity level of the RcsCD RcsB phosphorelay required for virulence. Expression of the $f l h D$-uidA and $f$ tsA-uidA fusions was measured just before inoculation $(0 \mathrm{~h})$ and $24 \mathrm{~h}$ and $48 \mathrm{~h}$ after inoculation in planta in wildtype and $r c s B$ genetic background (Fig. 4C). The ftsA-uidA fusion was expressed at a similar and very low level while the $f l h D$-uidA fusion was expressed at a similar and high level during all the virulence process, in both genetic backgrounds and at each time tested. A western blot analysis was performed with bacteria grown in planta. Similar amount of the RcsB protein was observed after $0 \mathrm{~h}, 24 \mathrm{~h}$ and $48 \mathrm{~h}$ of maceration (data not shown). Thus, these results indicate that the RcsCD RcsB phosphorelay activity is severely repressed during the virulence process in $D$. dadantii.

OPGs are one of the actors required for modulation of the RcsCD RcsB phosphorelay activity by medium osmolarity

OPGs synthesis and RcsCD RcsB activation are regulated by medium osmolarity in an opposite way: OPG concentration decrease (Cogez et al., 2001) while the RcsCD RcsB phosphorelay activation occurs as the medium osmolarity increases. Thus, the RcsCD RcsB activation could be modulated by osmolarity via variation of OPGs concentration. To test this 
1 hypothesis, motility was evaluated on swimming plates and the flhD-uidA and the ftsA-uidA

transcriptional fusion activities were determined in different genetic backgrounds at various osmolarities in M63 medium containing as a carbon source. In the wild-type strain (EC3937), motility remained constant in half strength M63 medium and in M63 medium then decreased as the osmolarity of the medium increased (Fig 5A). In the different mutant strains, motility levels remained unaffected whatever the osmolarity: as expected, a loss of motility was observed for the opgG strain (NFB3723) and motility similar to the wild-type strain was observed for the $r c s B$ (NFB7279), the $r c s B_{\mathrm{D} 56 \mathrm{~N}}(\mathrm{NFB7112})$ and the $r c s C$ (NFB3682) strains (Fig. 5A). The expression of the flhD-uidA fusion harbored by the wild-type strain (NFB3800) remained constant in half strength M63 medium and in M63 medium then decreased as the osmolarity of the medium increased (Fig. 6B). As expected, expression of the flhD-uidA fusion in the opgG (NFB7301) strain remained low and constant whatever the osmolarity while the expression level of the $f l h D$-uidA fusion harbored by the $\operatorname{rcs} B$ (NFB7202), the $r c s B_{\mathrm{D} 56 \mathrm{~N}}(\mathrm{NFB7399)}$ and the $\operatorname{rcs} C$ (NFB7128) strains was similar to the wild-type strain grown in M63 medium and remained unaffected when osmolarity was increased (Fig. 5B). These results indicate that repression of motility by medium osmolarity depends on variation of concentration of OPGs via activation of the RcsCD RcsB phosphorelay. For the ftsA-uidA transcriptional fusion activity (Fig. 7A), a low level of expression in the $\operatorname{rcsB}$ (NFB7205), the $r c s B_{\mathrm{D} 56 \mathrm{~N}}(\mathrm{NFB7408)}$ and the $\operatorname{rcs} C$ (NFB7127) strains was observed, whatever the osmolarity. Surprisingly, no increase in the expression of the ftsA-uidA fusion was observed in the wildtype strain (NFB3809). In the opgG strain (NFB7274), expression of the $f t s A$-uidA fusion was strongly increased as compared to the wild type strain and a 4 fold decrease was observed between the medium of lower osmolarity (half strength M63 medium) as compared to the medium of higher osmolarity (M63 full strength medium with $0.2 \mathrm{M} \mathrm{NaCl}$ added). This suggests that in addition to OPGs, additional factors are required for regulation of the ftsAZ 
1 operon by the RcsCD RcsB phosphorelay. As a control, OPGs concentrations were

2 determined (Fig. 6B). As previously shown (Cogez et al., 2001), OPGs concentration decreased as the osmolarity increased from $36 \mu \mathrm{g}$ of OPGs per mg of protein in half strength M63 medium to $6 \mu \mathrm{g}$ of OPGs per mg of protein in M63 medium with $0.2 \mathrm{M} \mathrm{NaCl}$ added. A western blot analysis was performed in $\mathrm{M} 63$ medium with $(0.2 \mathrm{M})$ or without $\mathrm{NaCl}$ added in an $\operatorname{opg} G, r \operatorname{cs} C, r \operatorname{cs} B_{\mathrm{D} 56 \mathrm{~N}}$ and in the wild-type strains (Fig. 3C). Similar amounts of the RcsB protein were observed whatever the strain and the osmolarity tested (data not shown). These results indicate that in $D$. dadantii, variation of OPGs concentration is required for proper activation of the RcsCD RcsB phosphorelay by osmolarity, but osmoregulation is a complex multifactor phenomenon (Wood, 1999) where OPGs are needed but are only one of the actors required for regulation of the $\mathrm{Rcs} C \mathrm{D} \operatorname{RcsB}$ regulon.

\section{Discussion}

OPGs are known to be an important virulence factor for many Gram-negative bacteria (Bohin, 2006) and we show in a previous paper (Bouchart et al., 2010) that OPGs control the RcsCD RcsB phosphorelay. In this paper we demonstrate that in addition concentration of OPGs in the periplasm modulates activation level of the RcsCD RcsB phosphorelay in D. dadantii.

In $D$. dadantii, motility and the $f l h D C$ operon are repressed by the activation of the RcsCD RcsB phosphorelay while the ftsA gene is induced (Bouchart et al., 2010) and reflect the RcsCD RcsB phosphorelay activation level. When OPG concentration depends on Larabinose concentration of the medium, we showed that motility and expression of both fusions genes tested depends on L-arabinose in an RcsB dependent manner and that activation of the RcsCD RcsB phosphorelay increase as the concentration of OPGs decrease. OPGs control the RcsCD RcsB activity in a concentration dependent manner. One of the stimuli activating the RcsCD RcsB phosphorelay is osmolarity increased (Majdalani et al., 2005) while in the same conditions, OPGs concentration decreased (Cogez et al., 2001; Bohin et al., 
1 2006). Decreased motility and expression of the flhD-uidA gene fusion observed when

2 osmolarity of the medium increased are in agreement with an increased activation of the

3 RcsCD RcsB phosphorelay and this activation depends both on a decreased OPG concentration and on RcsB (Fig. 5 and Fig. 6). No increase in the ftsA-uidA expression was observed when osmolarity increase while expression of this gene is known to be enhanced under activation of the RcsCD RcsB phosphorelay. One explanation is the presence, in $D$. dadantii, of a still unknown co-regulator of RcsB affecting interaction between RcsB and its DNA regulatory sequences. This hypothesis was strengthen by the decrease expression of the ftsA-uidA fusion gene in the opgG mutant strain since without additional regulatory component, the constitutive activation of the RcsCD RcsB phosphorelay would lead to a constant and high level of expression of this fusion. In E. coli, RcsA, GadE or BglJ form heterodimer with RcsB and co-regulate different subset of genes of the RcsCD RcsB regulon (Majdalani et al., 2005; Krin et al., 2010; Stratmann et al., 2012) but we did not found ortholog of these genes in the $D$. dadantii genome.

Variation in motility and in expression of target fusion genes is directly related the modulation of the RcsB phosphorylation level itself by RcsC since i/ no variation in the expression of both target genes was observed whatever the $\operatorname{rcs} B$ or $r \operatorname{cs} C$ mutant strains mutant strains and ii/ no difference in RcsB amount was observed in western blot analysis (see Fig. 3) whatever the growth conditions (data not shown). No difference in expression of the $r \operatorname{cs} B$ gene was also reported in Erwinia amylovora grown either in minimal medium or in planta (Wang et al., 2012). Thus, the activation level of the RcsC sensor and subsequently the phosphorylation level of RcsB depend on periplasmic concentration of OPG.

A minimal concentration of OPGs is required for initiating virulence (Fig. 3). This concentration could be estimated at $10 \mu \mathrm{g}$ of OPGs per mg of protein (Fig. 1) and was measured in M63 medium containing $0.01 \mathrm{~g} \mathrm{~L}^{-1}$ arabinose corresponding to the amount found 
1 in M63 medium supplemented with $0.15 \mathrm{M} \mathrm{NaCl}$ (fig. 6). This concentration of OPGs may correspond to the higher level of phosphorylated RcsB allowing virulence. Full virulence was observed until $20 \mu \mathrm{g}$ per mg of proteins and was measured in M63 medium containing $1 \mathrm{~g} \mathrm{~L}^{-1}$ arabinose or in M63 medium (fig. 6). No difference was observed in virulence (timing or severity of the disease) whatever the osmolarity of the medium before inoculation in planta. Our data indicate that $D$. dadantii may adjust its concentration of OPGs within the 24 first hours of infection. This concentration of OPGs was two times higher than the minimum required to ensure a low level of phosphorylated RcsB (Fig. 4). This high OPGs concentration may be explained by the low osmolarity of the plant apoplast even if this low osmolarity varies depending on the plant tissue (Boyer et al., 2001). Another explanation is that high OPGs are required for other cellular processes (Delangle et al., 2007) during virulence and that additional regulation enhances OPGs synthesis. In E. coli, the opgGH operon is part of the envelope stress sigma E factor regulon (Dartigalongue et al., 2001). In Yersinia enterocolitica, it was reported that the opgGH operon was induced within the host (Young and Miller, 1997). Thus, because OPGs are needed for virulence in several mammalian pathogenic enterobacteria, it would be of interest to measure OPG concentration in these bacterial species since in the gut high osmolarity environmental condition, high concentration of OPGs cannot be achieved by osmoregulation.

\section{Experimental procedures}

\section{Bacterial strains, media, antibiotics and growth condition}

Bacterial strains and plasmids are described in Table 1 . Bacteria were grown at $30^{\circ} \mathrm{C}(D$. dadantii) or $37^{\circ} \mathrm{C}$ (E. coli) in Lysogeny broth (LB) (Bertani, 2004), or in minimal medium M63 supplemented with glycerol as a carbon source at a concentration of $2 \mathrm{~g} \mathrm{l}^{-1}$ (Miller, 1992). Solid media were obtained by adding agar at $15 \mathrm{~g} \mathrm{l}^{-1}$. Osmolarity of the M63 medium 
1 increased by adding $0.1 \mathrm{M}$ or $0.2 \mathrm{M} \mathrm{NaCl}$ or decreased by a two-fold dilution. When necessary L(+)-arabinose (Calbiochem) was added in the M63 medium at indicated concentrations. X-Gluc (5-bromo-4-chloro-3-indolyl- $\beta$-D-glucuronide) was used in solid media at a concentration of $40 \mu \mathrm{g} \mathrm{ml}^{-1}$. Motility was tested on M63 agar plates containing $4 \mathrm{~g}$ $\mathrm{l}^{-1}$ agar and swim diameters were measured after $30 \mathrm{~h}$ of incubation.

Antibiotics were used at the following concentrations: gentamicin at $3 \mu \mathrm{g} \mathrm{ml}^{-1}$ (E. coli) or 2 $\mu \mathrm{g} \cdot \mathrm{ml}^{-1}$ (D. dadantii), chloramphenicol and tetracycline at $25 \mu \mathrm{g} \mathrm{ml}{ }^{-1}$ (E. coli) or $12.5 \mu \mathrm{g} \mathrm{ml}^{-1}$ (D. dadantii), ampicillin and kanamycin at $50 \mu \mathrm{g} \mathrm{m}^{-1}$ (E. coli) or $25 \mu \mathrm{g} \mathrm{ml}^{-1}$ (D. dadantii), streptomycin and spectinomycin at $100 \mu \mathrm{g} \mathrm{ml}^{-1}$ (E. coli) or $50 \mu \mathrm{g} \mathrm{ml}^{-1}$ (D. dadantii).

\section{Cloning of the opgGH operon under control of the araBAD promoter}

Plasmids and primers designed for PCR are listed in Table 1. The beginning of the operon opgGH (without the opgGH promoter) was amplified by PCR (opgGHnheIf and opgGHkpnIr primers) from pNFW32 (Page et al., 2001). This PCR product was digested by NheI and KpnI and cloned into pBAD18 digested by the same enzymes (pNFW208). pNFW32 was digested by EcoRI and KpnI and the DNA fragment containing the remaining of the opgGH operon was cloned into pNFW208 digested by the same enzymes. The resulting plasmid (pNFW212) contains the opgGH operon controlled by the $\operatorname{araBAD}$ promoter $\left(P_{B A D}\right.$ opgGH$)$. Plasmid pNFW212 was digested by AleI and Bstz17I and the DNA fragment containing the araC gene and $P_{B A D}$ opgGH operon was inserted in the SmaI site of pUC18Not. The resulting plasmid (pNFW219) was digested by NotI and the DNA fragment containing the araC gene and $P_{B A D}$ Opg $G H$ operon was inserted into the NotI site of pUTminiTn5-Spe. The resulting plasmid (pNFW233) was introduced in $D$. dadantii by conjugation to allow integration, after transposition of the miniTn5, of a single ectopic copy of the $\operatorname{araC}$ gene and $P_{B A D O p g G H}$ operon.

\section{Construction of the mutations}


1 The $\operatorname{rcs} B:: \mathrm{Gm}$ allele was constructed by excision of the $\mathrm{Cml}^{\mathrm{r}}$ cassette from the pNFW397

2 (Bouchart et al., 2010) digested by HpaI and replaced by the $\mathrm{Gm}^{\mathrm{r}}$ cassette digested by the

same restriction enzyme after PCR amplification (GmhpaIf and GmhpaIr, Table 1). The resulting plasmid (pNFW402) was introduced in D. dadantii cells by electroporation.

The $\operatorname{rcsB}_{\mathrm{D} 56 \mathrm{~N}}$ allele was constructed by SOE (splicing by overlap extension) PCR. The upstream region was amplified using primers RcsBDN3 and RcsBDNsoe1 (table 1), whereas the downstream region was amplified using primers RcsBDNsoe2 and RcsBDN4 (Table 1). The two PCR products were spliced together using the flanking primers RcsBDN3 and RcsBDN4. The mutagenesis PCR product was purified, digested with HpaI and AscI, cloned into pNFW257 digested by the same enzymes (pNFW374) and sequenced to confirm mutation. The NotI fragment from pNFW374, containing the $\operatorname{rcs} C B_{D 56 N} D$ locus, was subcloned into pUTmini-Tn5-Tet or pUTmini-Tn5-Spe digested by NotI. The resulting plasmids (pNFW379 and pNFW380 respectively) were introduced into D. dadantii cells by conjugation.

Transduction, conjugation and transformation.

Transformation of E. coli cells was carried out by the rubidium chloride technique (Miller, 1992). Plasmids were introduced in $D$. dadantii by electroporation (Sambrook et al., 1989) or conjugation (Miller, 1992). The insertions were integrated into the D. dadantii chromosome by marker exchange recombination in the presence of the appropriate antibiotic, after successive cultures in low phosphate medium (Roeder et al., 1985). Transduction with phage $\varphi \mathrm{EC} 2$ was carried out according to Resibois et al. (Resibois et al., 1984).

\section{Transposon mutagenesis}

To allow integration, after transposition of the miniTn5, of a single ectopic copy of the araC gene and $P_{B A D}$ opgGH operon or the $\operatorname{rcs} C D B_{\mathrm{D} 56 \mathrm{~N}}$ locus, transposon mutagenesis by mini-Tn5 was performed as described in (Bouchart et al., 2010). Briefly, after conjugation between an $E$. 
1 coli strain harboring the plasmid carrying the appropriate mini- $\operatorname{Tn} 5$ and a $D$. dadantii strain, mutants were selected on M63 plates containing sucrose as a unique carbon source and the required antibiotic.

\section{Determination of enzyme activities}

$\beta$-glucuronidase assays were performed on crude extracts obtained from bacteria disrupted by sonication 2x $20 \mathrm{~s}$ (Sonifier cell disruptor B-30, Branson, 70\% duty cycle, 7 microtip limit, Hold time, continuous, appropriate probe). $\beta$-glucuronidase activity was determined by spectrometric monitoring the hydrolysis of PNPU (4-nitrophenyl- $\beta$-D-glucuronide) at $405 \mathrm{~nm}$. The protein concentration was determined by the Bradford assay with bovine serum albumin as standard (Bradford, 1976).

OPGs analysis

Bacteria were grown in planta or in M63 medium (200 ml) supplemented either with various L-arabinose concentrations or with various concentrations of $\mathrm{NaCl}$ until mid-log phase. Bacteria were collected by centrifugation at $4^{\circ} \mathrm{C}$ for $15 \mathrm{~min}$ at $8,000 \times$ g. Cell pellets were resuspended in $20 \mathrm{ml}$ of distilled water and extracted with 5\% trichloroacetic acid (TCA). The TCA extracts were neutralized with ammonium hydroxide $10 \%$ and concentrated by rotary evaporation. The resulting material $(2 \mathrm{ml})$ was then fractionated by gel filtration on a Bio-Gel P-4 (Bio-Rad) column (55 by $1.6 \mathrm{~cm}$ ) equilibrated with $0.5 \%$ acetic acid. The column was eluted in the same buffer at a flow rate of $15 \mathrm{ml} \mathrm{h}^{-1}$, and fractions of $1.5 \mathrm{ml}$ were collected. Presence of sugar in each fraction was determined colorimetrically by the anthrone reagent procedure. Fractions containing OPGs were pooled and content was determined by the same procedure (Spiro, 1966). For mass spectrometry analysis, OPGs were subsequently desalted in water on a Bio-Gel P-2 (Bio-Rad) column (70 by $1.6 \mathrm{~cm})$ and fractions containing OPGs were lyophilized.

\section{Preparation of polyclonal antibodies against $\operatorname{Res} B$}


1 A DNA fragment encoding the $\operatorname{rcs} B$ gene of $D$. dadantii was amplified by PCR using the

2 primers rcsBacf and rcsBacr. The PCR product was cloned into a His ${ }_{6}$ tag expression vector, pET100/D-Topo® (Invitrogen Life Technologies) to give pNFW410. The resulting Histagged RcsB was expressed in E. coli BL21(DE3) and the protein was purified by affinity chromatography according to the manufacturer's procedure (Ni-nitrilotriacetic acid [NTA] agarose; Qiagen). The purified RcsB was used to immunize rabbit.

\section{Western blot hybridization}

Strains of $D$. dadantii were grown at $30^{\circ} \mathrm{C}$ to mid-log phase in M63 or after various time of infection in planta. Bacteria were collected by centrifugation and immediately lysed with SDS-PAGE buffer (2\% SDS, 2.5\% DTT, $25 \mathrm{mM}$ Tris- $\mathrm{HCl} \mathrm{pH}$ 7.5, 15\% glycerol), heated to $100^{\circ} \mathrm{C}$ for $5 \mathrm{~min}$ and loaded onto a $10 \%$ SDS-polyacrylamide gel (34). Proteins from the gel were transferred onto a nitrocellulose membrane (Whatman). Western blotting was performed using standard protocols with the rabbit anti-RcsB polyclonal antibodies at a dilution of 1:1000 and anti-rabbit secondary antibody coupled to horseradish peroxidase (dilution 1:10,000). Blots were imaged by chemiluminescent detection (ECL kit, GE healthcare Pharmacia Biotech).

\section{Pathogenicity test}

Chicory leaves were inoculated as previously described (Delangle et al., 2007; Bouchart et al., 2010).

\section{Bacteria isolation from infected chicory leaves}

Infected chicory leaves were introduced into tubes (one leave per tube) containing $20 \mathrm{~mL}$ of minimal medium 63 without carbon source added. Bacteria were suspended in the medium by vigorous shaking of the tubes during $15 \mathrm{~min}$. Leaves were then carefully removed from the tube. The medium was filtered through a paper filter (previously sterilized) to separate few macerated tissue release during the shaking step from bacteria (no more than $6 \%$ bacteria 
1 were lost during this step). Bacteria were then collected by centrifugation at $4^{\circ} \mathrm{C}$ for $15 \mathrm{~min}$ at $28,000 \times$ g. Cell pellets were resuspended in $20 \mathrm{~mL}$ of minimal medium 63 for OPGs analysis

or in $2 \mathrm{~mL}$ of the same medium for $\beta$-glucuronidase assays. Before analysis or assays, an aliquot of $0.1 \mathrm{~mL}$ of each bacterial suspension was taken up, diluted and counted. A total of $10^{8}$ to $10^{10} \mathrm{CFU}$ were recovered per chicory leave depending on the incubation time.

Matrix-assisted laser desorption/ionization mass spectrometry (MALDI-MS)

All mass spectra were acquired on a Voyager Elite (DE-STR) reflectron time-of-flight (TOF) mass spectrometer (Perseptive Biosystems, Framingham, MA), equipped with a pulsed nitrogen laser $(337 \mathrm{~nm})$ and a gridless delayed extraction ion source. Oligosaccharide samples were analyzed in delayed extraction mode using an accelerating voltage of $20 \mathrm{kV}$, a pulse delay time of $200 \mathrm{~ns}$ and a grid voltage of $66 \%$. Detector bias gating was used to reduce the ion current for masses below $500 \mathrm{Da}$. Between 100 and 200 scans were averaged for each mass spectrum. Oligosaccharide alditols were co-crystallized with 2,5-dihydroxybenzoic acid (DHB) as matrix $\left[10 \mathrm{mg} \mathrm{ml}^{-1}\right.$ of DHB in methanol/water (50/50) containing $0.1 \%$ Trifluoro Acetic acid (TFA)]. For all measurements, the "dried droplet" preparation technique was used. Typically, $1 \mu \mathrm{l}$ of the matrix was mixed on-target with $1 \mu \mathrm{l}$ of water-dissolved oligosaccharides and allowed to dry under an air stream. They were analyzed in positive ion mode.

\section{Acknowledgements}

This work was supported by grants from the Centre National de la Recherche Scientifique (CNRS) and from the Ministère de l'Education Nationale et de la Recherche.

\section{References}

Bertani, G. (2004) Lysogeny at Mid-Twentieth Century: P1, P2, and Other Experimental Systems. J Bacteriol 186: 595-600. 
1 Bhagwat, A.A., Jun, W., Liu, L., Kannan, P., Dharne, M., Pheh, B., et al. (2009)

2 Osmoregulated periplasmic glucans of Salmonella enterica serovar Typhimurium are required 3 for optimal virulence in mice. Microbiology 155: 229-237.

4 Bohin, J.P., and Lacroix, J.M. (2006) Osmoregulation in the periplasm. In The periplasm.

5 Ehrmann, M. (ed) Washington, DC: American Society for Microbiology, pp 325-341.

6 Bouchart, F., Delangle, A., Lemoine, J., Bohin, J.P., and J.M. (2007) Proteomic analysis of a non virulent mutant of the phytopathogenic bacterium Erwinia chrysanthemi deficient in osmoregulated periplasmic glucans: change in protein expression is not restricted to the envelope, but affects general metabolism. Microbiology 153: 760-767.

Bouchart, F., Boussemart G., Prouvost A.F., Cogez V., Madec E., Vidal O., et al. (2010) The virulence of a Dickeya dadantii 3937 mutant devoid of osmoregulated periplasmic glucans (OPGs) is restored by inactivation of the RcsCD RcsB phosphorelay. J Bacteriol 192: 34843490 .

Boyer, J.S. (2001) Growth-induced water potentials originate from wall yielding during growth. J Exp Bot 52: 1483-1488.

Bradford, M.M. (1976) A rapid and sensitive method for the quantitation of microgram quantities of protein utilizing the principle of protein-dye binding. Anal Biochem 72: 248-254. Buelow, D.R., and Raivio, R. (2010) Three (and more) component regulatory systems auxiliary regulators of bacterial histidine kinases. Mol Microbiol 75: 547-566.

Cano, D.A., Dominguez-Bernal, G., Tierrez, A., Garcia-Del Portillo, F., and Casadesus, J. (2002) Regulation of capsule synthesis and cell motility in Salmonella enterica by the essential gene igaA. Genetics 162: 1513-1523.

Carballès, F., Bertrand, C., Bouché, J.P., and Cam, K. (1999) Regulation of Escherichia coli cell division genes $f t s A$ and $f t s Z$ by the two-component system RcsC-RcsB. Mol Microbiol 34: 442-450.

Cogez, V., Talaga, P., Lemoine, J., and Bohin, J.P. (2001) Osmoregulated periplasmic glucans of Erwinia chrysanthemi. J bacteriol 183: 3127-3133.

Dartigalongue, C., Missiakas, D., and Raina, S. (2001) Characterization of the Escherichia coli sigma E regulon. J biol chem 276: 20866-20875.

De Lorenzo, V., and Timmis, K.N. (1994) Analysis and construction of stable phenotypes in Gram-negative bacteria with Tn5- and Tn10-derived minitransposons. Methods Enzymol 235: 386-405. 
1 De Lorenzo, V., Herrero, M., Jakubzik, U., and Timmis, K.N. (1990) Mini-Tn5 transposon derivatives for insertion mutagenesis, promoter probing, and chromosomal insertion of cloned DNA in gram-negative eubacteria. J Bacteriol 172: 6568-6572.

Delangle, A., Prouvost, A.F., Cogez, V., Bohin, J.P., Lacroix, J.M., and Hugouvieux-CottePattat, N. (2007) Characterization of the Erwinia chrysanthemi gan locus involved in galactan catabolism. J Bacteriol 189: 7053-7061.

Francez-Charlot, A., Laugel, B., Van Gemert, A., Dubarry,N., Wiorowski, F., CastaniéCornet, M.P., et al. (2003) RcsCDB His-Asp phosphorelay system negatively regulates the flhDC operon in Escherichia coli. Mol Microbiol 49: 823-832.

Garcia-Calderon, C.B., Garcia-Quintanilla, M., Casadesus, J., and Ramos-Morales, F. (2005) Virulence attenuation in Salmonella enterica $\operatorname{rcs} C$ mutants with constitutive activation of the Rcs system. Microbiology 151: 579-588.

Guzman, L.M., Belin, D., Carson, M.J., and Beckwith, J. (1995) Tight regulation, modulation, and high-level expression by vectors containing the arabinose PBAD promoter. J Bacteriol 177: 4121-4130.

Hagiwara, D., Sugiura, M., Oshima, T., Mori, H., Aiba, H., Yamashino, T., and Mizuno, T. (2003) Genome-wide analyses revealing a signaling network of the RcsC-YojN-RcsB phosphorelay system in Escherichia coli. J bacteriol 185: 5735-5746.

Krin, E., Danchin, A., and Soutourina, O. (2010) RcsB plays a central role in H-NSdependent regulation of motility and acid stress resistance in Escherichia coli. Res microbiol 161: 363-371.

Latasa, C., Garcia, B., Echeverz, M., Toledo-Arana, A., Valle, J., Campoy, S., et al. (2012) Salmonella biofilm development depends on the phosphorylation status of RcsB. J Bacteriol 194: 3708-3722.

Loubens, I., Debarbieux, L., Bohin, A., Lacroix, J.M., and Bohin, J.P. (1993) Homology between a genetic locus $(m d o A)$ involved in the osmoregulated biosynthesis of periplasmic glucans in Escherichia coli and a genetic locus ( $h r p M)$ controlling pathogenicity of Pseudomonas syringae. Mol Microbiol 10: 329-340.

Mahajan-Miklos, S., Tan, M.W., Rahme, L.G., and Ausubel, F.M. (1999) Molecular mechanisms of bacterial virulence elucidated using a Pseudomonas aeruginosaCaenorhabditis elegans pathogenesis model. Cell 96: 47-56.

Majdalani, N., and Gottesman, S. (2005) The Rcs phosphorelay: a complex signal transduction system. Annu Rev Microbiol 59: 379-405. 
1 Miller, J.H. (1992) A short course in bacterial genetics: a laboratory manual and handbook for 2 Escherichia coli and related bacteria. New York USA Cold Spring Harbor Laboratory Press.

3 Mouslim, C., Latifi, T., and Groisman, E.A. (2003) Signal-dependent requirement for the coactivator protein RcsA in transcription of the RcsB-regulated $u g d$ gene. $\mathrm{J}$ of biol chem 278 : 50588-50595.

Page, F., Altabe, S., Hugouvieux-Cotte-Pattat, N., Lacroix, J.M., Robert-Baudouy, J., and Bohin, J.P. (2001) Osmoregulated periplasmic glucan synthesis is required for Erwinia chrysanthemi pathogenicity. J Bacteriol 183: 3134-3141.

Perombelon, M.C.M. (2002) Potato diseases caused by soft-rot erwinias : an overview of pathogenesis. Plant Pathol 51: 1-12.

Resibois, A., Colet, M., Faelen, M., Schoonejans, T., and Toussaint, A. (1984) Phi-EC2, a new generalized transducing phage of Erwinia chrysanthemi. Virology 137: 102-112.

Roeder, D.L., and Collmer, A. (1985) Marker-exchange mutagenesis of a pectate lyase isozyme gene in Erwinia chrysanthemi. J Bacteriol 164: 51-56.

Sambrook, J., Fritsch, E.F., and Maniatis, T. (1989) Molecular cloning: a laboratory manual 2nd edn. New York USA, Cold Spring Harbor Laboratory press.

Sledjeski, D.D., and Gottesman, S. (1996) Osmotic shock induction of capsule synthesis in Escherichia coli K-12. J bacteriol 178: 1204-1206.

Spiro, R.G. (1966) Analysis of sugars found in glycoproteins. Methods Enzymol 8: 3-27.

Stratmann, T., Pul, U., Wurm, R., Wagner, R., and Schnetz, K. (2012) RcsB-BglJ activates the Escherichia coli leuO gene, encoding an H-NS antagonist and pleiotropic regulator of virulence determinants. Mol microbiol 83: 1109-1123.

Vieira, J., and Messing, J. (1982) The pUC plasmids, an M13mp7-derived system for insertion mutagenesis and sequencing with synthetic universal primers. Gene 19: 259-268.

Wang, D., Qi, M., Calla, B., Korban, S.S., Clough, S.J., Cock, P.J. et al. (2012) Genome-wide identification of genes regulated by the Rcs phosphorelay system in Erwinia amylovora. Mol plant-microbe interact 25: 6-17.

Wood, J.M. (1999) Osmosensing by bacteria: signals and membrane-based sensors. Microbiol Mol biol rev 63: 230-262.

Yanisch-Perron, C., Vieira, J., and Messing, J. (1985) Improved M13 phage cloning vectors and host strains: nucleotide sequences of the M13mp18 and pUC19 vectors. Gene 33: 103119.

Young, G.M., and Miller, V.L. (1997) Identification of novel chromosomal loci affecting Yersinia enterocolitica pathogenesis. Mol microbiol 25: 319-328. 
Source or reference primer

D. dadantii

\begin{tabular}{|c|c|c|}
\hline EC3937 & Wild type & Laboratory collection \\
\hline NFB3723 & $o p g G:: \mathrm{Cml}$ & Laboratory collection \\
\hline NFB3682 & $\mathrm{rcs} C:: \mathrm{Cml}$ & Bouchart et al., 2010 \\
\hline NFB3800 & flhD-uidA-Kan ${ }^{r}$ & Bouchart et al., 2010 \\
\hline NFB3809 & ftsA-uidA-Kan ${ }^{r}$ & Bouchart et al., 2010 \\
\hline NFB3835 & $o p g G:: C m l, \mathrm{P}_{\mathrm{BAD}} O p g G H-\mathrm{Spe}^{\mathrm{r}}$ & This study \\
\hline NFB3837 & opgG::Cml, $\mathrm{P}_{\mathrm{BAD}}$ opgGH-Spe ${ }^{r}$, ftsA-uidA-Kan $^{r}$ & This study \\
\hline NFB7112 & $r c s B:: C m l, r_{D S C B} D-S p e^{r}$ & This study \\
\hline NFB7127 & $\operatorname{rcs} C:: C m l, f t s A-u i d A-K a n^{r}$ & Bouchart et al., 2010 \\
\hline NFB7128 & rcsC::Cml, flhD-uidA-Kan ${ }^{r}$ & Bouchart et al., 2010 \\
\hline NFB7202 & rcsB::Cml, flhD-uidA-Kan ${ }^{r}$ & Bouchart et al., 2010 \\
\hline NFB7205 & rcsB::Cml, ftsA-uidA-Kan ${ }^{\mathrm{r}}$ & Bouchart et al., 2010 \\
\hline NFB7274 & opgG::Cml, ftsA-uidA-Kan ${ }^{r}$ & This study \\
\hline NFB7279 & $r \operatorname{cs} B:: G m$ & This study \\
\hline NFB7300 & opgG::Cml, $\mathrm{P}_{\mathrm{BAD}}$ opgGH-Spe ${ }^{\mathrm{r}}$, flhD-uidA-Kan ${ }^{\mathrm{r}}$ & This study \\
\hline NFB7301 & opgG::Cml, flhD-uidA-Kan ${ }^{r}$ & This study \\
\hline NFB7368 & $o p g G:: C m l, r c s B:: G m, P_{\mathrm{BAD}} O p g G H-S_{p e}{ }^{r}$ & This study \\
\hline NFB7369 & opgG::Cml, rcsB::Gm, $\mathrm{P}_{\mathrm{BAD}} O p g G H-\mathrm{Spe}^{\mathrm{r}}$, flhD-uidA-Kan ${ }^{\mathrm{r}}$ & This study \\
\hline NFB7371 & opgG::Cml, rcsB::Gm, $\mathrm{P}_{\mathrm{BAD}} O p g G H-\mathrm{Spe}^{\mathrm{r}}$, ftsA-uidA-Kan ${ }^{\mathrm{r}}$ & This study \\
\hline NFB7399 & $r \operatorname{cs} B:: C m l, r c s C B_{D 56 N} D-S p e^{r}$, flhD-uidA-Kan ${ }^{r}$ & This study \\
\hline NFB7400 & opgG::FRT, rcsC::Cml, $\mathrm{P}_{\mathrm{BAD}} O p g G H-\mathrm{Spe}^{\mathrm{r}}$ & This study \\
\hline NFB7401 & 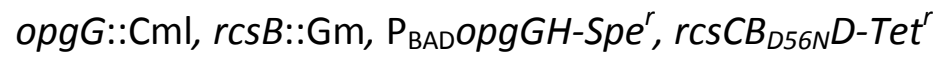 & This study \\
\hline
\end{tabular}


NFB7402

NFB7403

NFB7405

NFB7406

NFB7408

E. coli

BL21 (DE3)

JM83

S17- $\lambda$ pir

NFB3276

Plasmids

pUC18Not

pUTminiTn5-Spe

pUTminiTn5-Tet

pBAD18

pNFW32

pNFW208

pNFW212

pNFW219

pNFW257

pNFW233

pNFW374

pNFW379

pNFW380

pNFW397

pNFW402

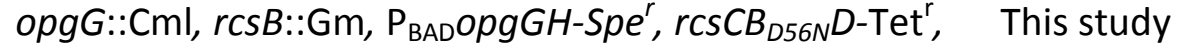
ftsA-uidA-Kan ${ }^{r}$

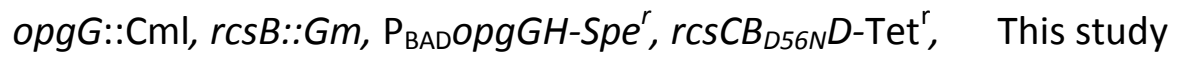
flhD-uidA-Kan ${ }^{\mathrm{r}}$

opgG::FRT, rCsC::Cml, $\mathrm{P}_{\mathrm{BAD}}$ OpgGH-Spe ${ }^{\mathrm{r}}$, ftsA-uidA-Kan ${ }^{\mathrm{r}} \quad$ This study

opgG::FRT, rcsC::Cml, $\mathrm{P}_{\mathrm{BAD} O} O p g G H-\mathrm{Spe}^{r}$, ftsA-uidA-Kan ${ }^{r} \quad$ This study

$\operatorname{rcs} B:: C m l, r \operatorname{rsC} B_{\mathrm{D} 56 \mathrm{~N}} D-\mathrm{Spe}^{\mathrm{r}}$, ftsA-uidA-Kan $^{\mathrm{r}} \quad$ This study

ompT, hsdSB, gal, dcm

ara $\Delta$ (lac-proAB), rpsL, thi, ( $\varphi 80 \Delta$ lacZ-M15)

recA1, thi, pro, hsdR-M+, RP4:2-Tc::Mu-Kan::Tn7, גpir

S17- $\lambda$ pir, pNFW233

Invitrogen

Vieira et al., 1982

De Lorenzo et al., 1994

This study

$A m p^{r}$

Yanisch-Perron et al.,

1985

De Lorenzo et al., 1990

De Lorenzo et al., 1990

Guzman et al., 1995

Page et al., 2001

This study

This study

This study

Bouchart et al., 2010

This study

This study

This study

This study

Bouchart et al., 2010

This study 
pNFW410

Primers

opgGHnhelf

opgGHkpnIr

gmhpalf

gmhpalr

rcsBacf

rcsBacr

rCsBDN3

rcsBDNsoe1

rcsBDNsoe2

rCsBDN4
pET100/D-topo rcsB

This study

Sequence ${ }^{c, d}$

GGGT GCTAGCTGATTTAGGCACACATACAGGGGG

AACTGGTACCAGACGGCAACGCCGTGTCGATAGCC

GTGTGTTAACCCCCATCCCCCTGTTGAC

GTGTGTTAACCGCAAGCTAGCTTGGCTGC

CACCATGAGCAATCTAAACGTAATTATTGCAG

CGCCGGCAAGAGCATTACTC

CTGAAAGGTGTCTTTGCCATGCTGAATCTTCATCCC

GCATGGATAGATTGGTGATCAGGAC

GTCCTGATCACCAATCTATCCATGC

TTCCAGCACTTTGCTGACGCTTTCC

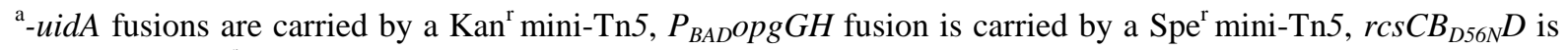
carried by a Tet ${ }^{r} \operatorname{mini}-\mathrm{Tn} 5$.

${ }^{\mathrm{b}} \mathrm{Cml}^{\mathrm{r}}$, chloramphenicol resistance; $A \mathrm{Am}^{\mathrm{r}}$, ampicillin resistance, $\mathrm{Kan}^{\mathrm{r}}$, kanamycin resistance, $\mathrm{Spe}^{\mathrm{r}}$, spectinomycin resistance, $\mathrm{Str}^{\mathrm{r}}$, streptomycin resistance, $\mathrm{Gm}^{\mathrm{r}}$, gentamicin resistance, Tet $^{\mathrm{r}}$, tetracycline.

${ }^{\mathrm{c}}$ Restriction sites are underlined. ${ }^{\mathrm{d}}$ The mutation is indicated in bold.

Figure legends

Fig. 1. OPGs concentration of the NFB3835 $\left(\mathrm{P}_{B A D O p g G H}\right)$ at various concentrations of Larabinose. Bacteria were grown until mid-log phase in M63 medium supplemented with indicated concentrations of L-arabinose. OPGs were extracted, purified and quantified as described in procedures. Results reported are the average of 3 independents experiments.

Fig. 2. Effect of the arabinose concentration on motility (A) and expression of the flhD-uidA semisolid plates supplemented with indicated L-arabinose concentrations. Swim diameters were measured after $30 \mathrm{~h}$ of incubation at $30^{\circ} \mathrm{C}$. For fusion expression measurements, bacteria 
were grown until mid log phase in the same media and broken by passing through a French pressure cell. The $\beta$-glucuronidase activities were measured with PNPU as a substrate. Specific activity is expressed as the change in optical density at $410 \mathrm{~nm}$ per min and per $\mathrm{mg}$ of protein. Results reported are the average of 3 independents experiments.

Fig. 3 Effect of arabinose concentration on pathogenicity of the NFB3835 ( $\mathrm{P}_{B A D}$ opgGH) strain. Bacteria were grown until mid log phase in M63 medium supplemented with indicated L-arabinose concentrations, washed twice and $10^{7}$ bacteria were inoculated into scarified chicory leaves. Evolution of the disease symptoms were observed after 24 and $48 \mathrm{~h}$.

Fig. 4. Effect of OPG concentration in planta on RcsCD RcsB activation. For determination of OPG concentration, mass spectra or fusion activities, bacteria were collected after growth to mid $\log$ phase in 63 medium $(0 \mathrm{~h})$, or after growth in planta during $24 \mathrm{~h}$, or after growth in planta during $48 \mathrm{~h}$. For each time, CFU were determined. A/ concentration of OPGs purified from the wild-type strain (EC3937) as described in experimental procedures. B/ MALDI mass spectra acquired in positive ion mode on purified OPGs extracted from the wild-type strain. The $[\mathrm{M}+\mathrm{Na}]^{+}$molecular ions are indicated on the top of each peak. Their respective $[\mathrm{M}+\mathrm{K}]^{+}$ molecular ions are identified by an asterisk and have an increment of $16 \mathrm{Da}$ in comparison to the corresponding $[\mathrm{M}+\mathrm{Na}]^{+}$ions. $\mathrm{C} /$ Expression of the flhD-uidA or the ftsA-uidA fusion genes in the wild-type (NFB3800 and NFB3809 respectively) or the $\operatorname{rcsB}$ (NFB7202 and NFB7205 respectively) strains. The $\beta$-glucuronidase activities were measured with PNPU as a substrate. Specific activity is expressed as the change in optical density at $410 \mathrm{~nm}$ per min and per $10^{9}$ bacteria. Results reported are the average of 3 independents experiments. 
1 Fig. 5 Effect of medium osmolarity on motility (A) and expression of the flhD-uidA (B) gene

2 fusion in various strains. Motility was measured in half strength M63, M63, M63 supplemented with $0.1 \mathrm{M}$ or $\mathrm{M} 63$ supplemented with $0.2 \mathrm{M} \mathrm{NaCl}$ semisolid plates. For fusion expression measurements, bacteria were grown until mid log phase in the same media and broken by passing through a French pressure cell. The $\beta$-glucuronidase activities were measured with PNPU as a substrate. Specific activity is expressed as the change in optical density at $410 \mathrm{~nm}$ per min and per $\mathrm{mg}$ of protein. Results reported are the average of 3 independents experiments.

Fig. $6 \mathrm{~A} /$ Effect of medium osmolarity on expression of the ftsD-uidA (A) gene fusion in various strains. For fusion expression measurements, bacteria were grown in half strength M63, M63, M63 supplemented with $0.1 \mathrm{M}$ or M63 supplemented with $0.2 \mathrm{M} \mathrm{NaCl}$ until mid$\log$ phase and broken by passing through a French pressure cell. The $\beta$-glucuronidase activities were measured with PNPU as a substrate. Specific activity is expressed as the change in optical density at $410 \mathrm{~nm}$ per min and per mg of protein. Results reported are the average of 3 independents experiments. B/ OPGs concentration of the wild-type strain (EC3937) at various osmolarities. Bacteria were grown until mid log phase in the same media. OPGs were extracted, purified and quantified as described in experimental procedures. Results reported are the average of 3 independents experiments. 